\title{
2.5-D modeling of cross-hole electromagnetic measurement by finite element method
}

\author{
Shen Jinsong ${ }^{1,2} *$ and Sun Wenbo ${ }^{1}$ \\ ${ }^{1}$ Department of Geophysics, School of Resources and Information Technology, China University of Petroleum, Beijing \\ 102249, China \\ ${ }^{2}$ CNPC Key Lab of Geophysical Exploration, China University of Petroleum, Beijing 102249, China
}

\begin{abstract}
A finite element method is developed for simulating frequency domain electromagnetic responses due to a dipole source in the 2-D conductive structures. Computing costs are considerably minimized by reducing the full three-dimensional problem to a series of two-dimensional problems. This is accomplished by transforming the problem into y-wave number $\left(K_{y}\right)$ domain using Fourier transform and the y-axis is parallel to the structural strike. In the $K_{y}$ domain, two coupled partial differential equations for magnetic field Hy and electric field Ey are derived. For a specific value of $K_{y}$, the coupled equations are solved by the finite element method with isoparametric elements in the $\mathrm{X}-\mathrm{z}$ plane. Application of the inverse Fourier transform to the $K_{y}$ domain provides the electric and magnetic fields in real space. The equations derived can be applied to general complex two-dimensional structures containing either electric or magnetic dipole source in any direction. In the modeling of the electromagnetic measurement, we adopted a pseudo-delta function to distribute the dipole source current and circumvent the problem of singularity at the source point. Moreover, the suggested method used isoparametric finite elements to accommodate the complex subsurface formation. For the large scale linear system derived from the discretization of the Maxwell's equations, several iterative solvers were used and compared to select the optimal one. A quantitative test of accuracy was presented which compared the finite element results with analytic solutions for a dipole source in homogeneous space for different ranges and different wave numbers $K_{y}$. to validate the code and check its effectiveness. In addition, we addressed the effects of the distribution range $\tau$ of the pseudo-delta function on the numerical results in homogeneous medium.
\end{abstract}

Key words: $2.5-\mathrm{D}$, electromagnetic modeling, frequency domain, isoparametric finite element, pseudodelta function

\section{Introduction}

Electromagnetic methods provide information about the formation resistivity which is one of the most important parameters in saturation evaluation in the oil industry (Farrelly et al, 2004). Forward modeling is one of the most common and effective methods that help us understand the physical significance of the electromagnetic responses. However, there are many restrictions on electromagnetic modeling which are caused by the difficulty of dealing properly with actual 2-D or 3-D subsurface structures and considerable numbers of inhomogeneities (Tompkins, 2004; Avdeeva et al, 2007). In order to simulate actual subsurface formation for surveys in complex geological areas, the configuration irregularity of the target formation must also be considered (Gallardo et and Meju, 2003), which becomes a difficult problem for traditional modeling methods, such as finite difference method. Moreover, we must be able to put inhomogeneities

*Corresponding author. email: shenjinsong@cup.edu.cn

Received June 28, 2007 near the source to estimate the effect of source overprint (Ueda and Zhdanov, 2005; Zaslavsky, 2006; Johansen et al, 2005).

In this study, we implemented the modeling of the socalled 2.5-D problem which considers a 3-D source over a 2-D earth (MacGregor et al, 2006). With respect to 2.5-D electromagnetic modeling, only a limited number of papers are published because of the difficulty of dealing with the source. Kong et al (2008) calculated the frequency domain responses excited by a magnetic dipole source with the finite element method (FEM). Leppin (1992) and Liu and Sato (2004) estimated the time domain responses generated by a rectangular current loop with the implicit Finite Difference Method (FDM) in a simple medium. The finite element method (FEM) was adopted by David and Morrison (1995) to compute the electromagnetic fields induced by a magnetic dipole source to simulate inhomogeneities of the formation. Sugeng et al (1996) also applied the isoparametric finite element method to determining the secondary electromagnetic fields excited by a rectangle loop.

In the published results, most of the schemes are carried 
out by solving the secondary field due to anomalous bodies (Davydycheva et al, 2003). The reason for isolating the primary field is that the dipole source term is singular at the source point and is difficult to be expressed accurately by discrete equations (Goldman et al, 1986). For most cases, the primary field is calculated for the homogeneous or 1-D structure for which analytical equations exist (Hoversten et al, 2003; Hoversten and Newman, 2004; 2006). This classical separation of the primary and the secondary field is not effective for complex subsurface formation because of the difficulty of determining the background structure (Hou and Torres-Verdín, 2003).

In this study, we derived the basic equations of the 2.5-D electromagnetic problem and used a pseudo-delta function to express a dipole source (Constable and Weiss, 2004; Constable, 2006; Constable and Srnka, 2007; Abubakar et al, 2006). We focused on the numerical considerations of finite element electromagnetic modeling. Several experiments were carried out to demonstrate the effect of the approximate pseudo-delta on the numerical accuracy and efficiency (Buell,1991). By using the approximate pseudodelta, the problems of separating the total electromagnetic fields into primary and secondary field and determination of the background structure were avoided. In addition, we could easily simulate the complex subsurface formation by using the isoparametric FEM. In order to check the validity and efficiency of the code, the numerical result was first compared with the analytic solution in the homogeneous structure with different linear system solvers. Then the layer model was used to probe the optimal wave number range of $K_{y}$. Furthermore, we addressed the effects of the distribution range $\tau$ of the pseudo-delta function on the numerical results in homogeneous medium. Finally, we focused attention on the cross-hole electromagnetic responses of the complex formation structures often encountered in oil industry.

\section{Basic equations of the 2.5-D electromagnetic problem}

The earth model considered in this paper is shown in Fig. 1, where the $y$-axis is set to be the strike direction. Conductivity $\sigma$, dielectric permittivity $\varepsilon$ and magnetic permeability $\mu$ are invariants along the $y$-axis and they vary only in the $x-z$ plane. The electric or magnetic dipole source can be located anywhere and in any direction. Assuming a time dependence function $e^{i w t}$, the electric field $E$ and magnetic field $H$ are described by Maxwell's equations

$$
\begin{gathered}
\nabla \times E=\hat{z} H-M_{s} \\
\nabla \times H=\hat{y} E+J_{s}
\end{gathered}
$$

where $M_{s}$ and $J_{\mathrm{s}}$ are the impressed magnetic and electric sources respectively, and the impedivity $\hat{z}=i \mu \omega$ and the admittivity $\hat{y}=\sigma+i \varepsilon \omega$ (Ward and Hohmann, 1988) are used.

After adoption of the curl operator, we have

$$
\begin{aligned}
& \left(\frac{\partial E_{z}}{\partial y}-\frac{\partial E_{y}}{\partial z}\right) \overrightarrow{e_{x}}+\left(\frac{\partial E_{x}}{\partial z}-\frac{\partial E_{z}}{\partial x}\right) \overrightarrow{e_{y}}+\left(\frac{\partial E_{y}}{\partial x}-\frac{\partial E_{x}}{\partial y}\right) \overrightarrow{e_{z}} \\
& =-\hat{z}\left[\left(H_{x}+M_{s x}\right) \overrightarrow{e_{x}}+\left(H_{y}+M_{s y}\right) \overrightarrow{e_{y}}+\left(H_{z}+M_{s z}\right) \overrightarrow{e_{z}}\right] \\
& \left(\frac{\partial H_{z}}{\partial y}-\frac{\partial H_{y}}{\partial z}\right) \overrightarrow{e_{x}}+\left(\frac{\partial H_{x}}{\partial z}-\frac{\partial H_{z}}{\partial x}\right) \overrightarrow{e_{y}}+\left(\frac{\partial H_{y}}{\partial x}-\frac{\partial H_{x}}{\partial y}\right) \overrightarrow{e_{z}} \\
& =\left(\hat{y} E_{x}+J_{s x}\right) \overrightarrow{e_{x}}+\left(\hat{y} E_{y}+J_{s y}\right) \overrightarrow{e_{y}}+\left(\hat{y} E_{z}+J_{s z}\right) \overrightarrow{e_{z}}
\end{aligned}
$$

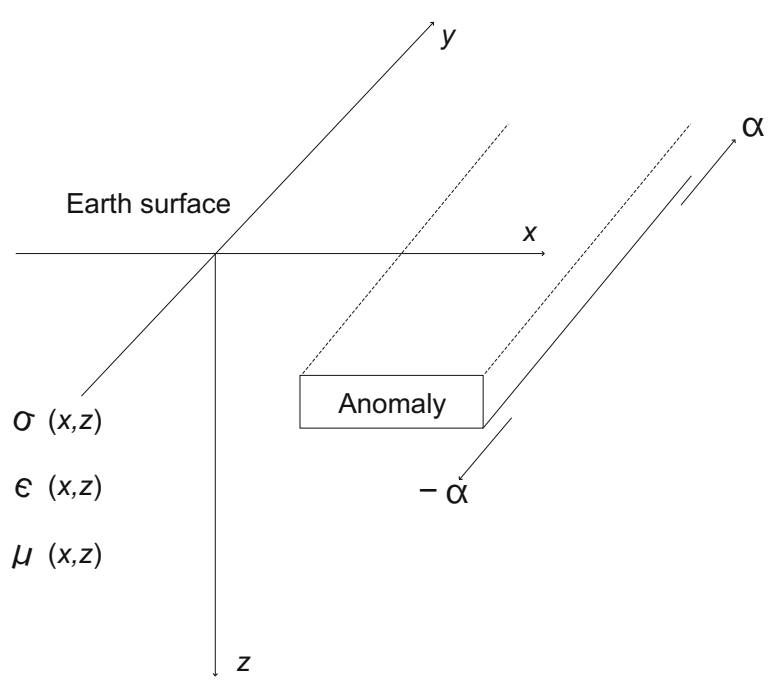

Fig. 1 Two-dimensional medium with y-axis strike

From equations (2a) and (2b), we can obtain six formulas of twelve variables on the electromagnetic field and its derivative.

Applying the Fourier transform to each component of $E$ and $H$ and source terms in equations (2a) and (2b) with respect to $y$, that is

$$
\widehat{F}\left(x, k_{y}, z, \omega\right)=\int_{-\infty}^{\infty} F(x, y, z, \omega) e^{-i k_{y} y} \mathrm{~d} y, \quad \frac{\partial \widehat{F}}{\partial y}=-i k_{y} \widehat{F}
$$

where $\widehat{F}$ can be $H_{x}, H_{y}, H_{z}, E_{x}, E_{y}$ or $E_{z}$.

We obtain two coupled governing differential equations for $\widehat{E_{y}}$ and $\widehat{H_{y}}$ (Stoyer and Greenfield, 1976).

$$
\begin{aligned}
& \frac{\partial}{\partial x}\left(\frac{\hat{y}}{k_{e}^{2}} \frac{\partial \widehat{E_{y}}}{\partial x}\right)+\frac{\partial}{\partial z}\left(\frac{\hat{y}}{k_{e}^{2}} \frac{\partial \widehat{E_{y}}}{\partial z}\right)-\hat{y} \widehat{E_{y}}+i k_{y}\left[\frac{\partial}{\partial x}\left(\frac{1}{k_{e}^{2}}\right) \frac{\partial \widehat{H_{y}}}{\partial z}\right. \\
&\left.-\frac{\partial}{\partial z}\left(\frac{1}{k_{e}^{2}}\right) \frac{\partial \widehat{H_{y}}}{\partial x}\right]= \widehat{J_{s y}}-i k_{y}\left[\frac{\partial}{\partial x}\left(\frac{1}{k_{e}^{2}} \widehat{J_{s x}}\right)+\frac{\partial}{\partial z}\left(\frac{1}{k_{e}^{2}} \widehat{J_{s z}}\right)\right] \\
&+\frac{\partial}{\partial x}\left(\frac{k^{2}}{k_{e}^{2}} \widehat{M_{s z}}\right)-\frac{\partial}{\partial z}\left(\frac{k^{2}}{k_{e}^{2}} \widehat{M_{s x}}\right)
\end{aligned}
$$




$$
\begin{aligned}
& \frac{\partial}{\partial x}\left(\frac{\hat{z}}{k_{e}^{2}} \frac{\partial \widehat{H_{y}}}{\partial x}\right)+\frac{\partial}{\partial z}\left(\frac{\hat{z}}{k_{e}^{2}} \frac{\partial \widehat{H_{y}}}{\partial z}\right)-\hat{z} \widehat{H_{y}}+i k_{y}\left[-\frac{\partial}{\partial x}\left(\frac{1}{k_{e}^{2}}\right) \frac{\partial \widehat{E_{y}}}{\partial z}\right. \\
&\left.+\frac{\partial}{\partial z}\left(\frac{1}{k_{e}^{2}}\right) \frac{\partial \widehat{E_{y}}}{\partial x}\right]= \hat{z} \widehat{M_{s y}}-i k_{y}\left[\frac{\partial}{\partial x}\left(\frac{\hat{z}}{k_{e}^{2}} \widehat{M_{s x}}\right)+\frac{\partial}{\partial z}\left(\frac{\hat{z}}{k_{e}^{2}} \widehat{M_{s z}}\right)\right] \\
&+\frac{\partial}{\partial x}\left(\frac{\hat{z}}{k_{e}^{2}} \widehat{J_{s z}}\right)-\frac{\partial}{\partial z}\left(\frac{\hat{z}}{k_{e}^{2}} \widehat{J_{s x}}\right)
\end{aligned}
$$

where $k^{2}=-\hat{z} \hat{y}$ and $k_{e}^{2}=k_{y}^{2}-k^{2}$.

The other components are obtained from the space derivatives of $E_{y}$ and $H_{y}$ as follows

$\widehat{E_{x}}=\frac{1}{k_{e}^{2}}\left(-i k_{y} \frac{\partial \widehat{E_{y}}}{\partial x}-\hat{z} \frac{\partial \widehat{H_{y}}}{\partial z}-\hat{z} \widehat{J_{s x}}-i k_{y} \hat{z} \widehat{M_{s z}}\right)$

$\widehat{E_{z}}=\frac{1}{k_{e}^{2}}\left(-i k_{y} \frac{\partial \widehat{E_{y}}}{\partial z}+\hat{z} \frac{\partial \widehat{H_{y}}}{\partial x}-\hat{z} \widehat{J_{s z}}-i k_{y} \hat{z} \widehat{M_{s x}}\right)$

$\widehat{H_{x}}=\frac{1}{k_{e}^{2}}\left(-i k_{y} \frac{\partial \widehat{H_{y}}}{\partial x}+\hat{y} \frac{\partial \widehat{E_{y}}}{\partial z}+k^{2} \widehat{M_{s x}}+i k_{y} \hat{z} \widehat{J_{s z}}\right)$

$\widehat{H_{z}}=\frac{1}{k_{e}^{2}}\left(-i k_{y} \frac{\partial \widehat{H_{y}}}{\partial z}-\hat{y} \frac{\partial \widehat{E_{y}}}{\partial x}+k^{2} \widehat{M_{s z}}-i k_{y} \hat{z} \widehat{J_{s x}}\right)$

\section{Finite element equations with isoparametric elements}

\subsection{Discretization of the Maxwell's equations}

In order to solve the above equations by the numerical method, we adopted the Galerkin method (Zienkiewicz, 1977). First, the calculation area was divided into isoparametric elements. Then, the higher order interpolation function of the finite element method was used to calculate the space derivative of $\widehat{E_{y}}$ and $\widehat{H_{y}}$ in equations. Here, a quadratic element with eight nodes which is shown in Fig.

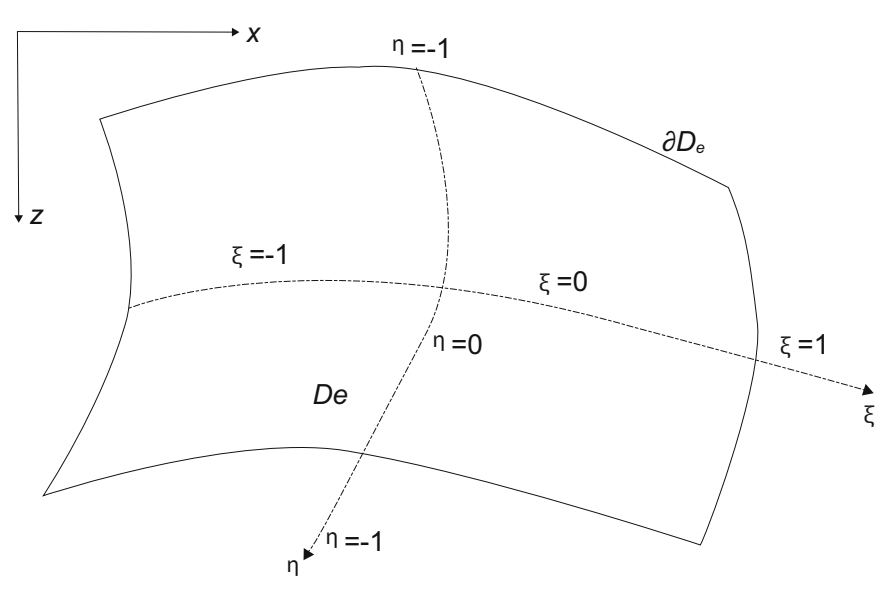

Fig. 2 Isoparametric finite element

2 was adopted. In each element, $\widehat{E_{y}}$ and $\widehat{H_{y}}$ are expressed by the quadratic interpolation function and the space derivative is able to be described by a linear interpolation function. Furthermore, in each element $\sigma, \mu, \varepsilon$ are constant and the interpolation function is represented by the local coordinates $\xi, \eta$ (Zienkiewicz, 1977).

The global coordinates and each component $F$ of electromagnetic field can be written as

$$
x=\sum_{j=1}^{8} N_{j}^{e} x_{j}, z=\sum_{j=1}^{8} N_{j}^{e} z_{j}, F=\sum_{j=1}^{8} N_{j}^{e} F_{j}
$$

$$
N_{i}^{e}= \begin{cases}\frac{1}{4}(1+\xi \xi)\left(1+\eta_{i} \eta\right)\left(\xi_{i} \xi+\eta_{i} \eta-1\right) & (i=1,3,5,7) \\ \frac{1}{2}\left(1-\xi^{2}\right)\left(1+\eta_{i} \eta\right) & (i=2,6) \\ \frac{1}{2}\left(1-\eta^{2}\right)\left(1+\xi_{i} \xi\right) & (i=4,8)\end{cases}
$$

where $N^{e}{ }_{i}$ is the quadratic interpolation function of the $i$-th node in the $e$-th element.

Applying the Galerkin method to equation (4a) and (4b), the linear equations system is obtained

$$
\begin{aligned}
& \sum_{e=1}^{N_{e}} \iint_{D_{e}}\left\{\frac{\partial N_{i}^{e}}{\partial x}\left(\frac{\hat{y}}{k_{e}^{2}} \frac{\partial \widehat{E_{y}}}{\partial x}\right)+\frac{\partial N_{i}^{e}}{\partial z}\left(\frac{\hat{y}}{k_{e}^{2}} \frac{\partial \widehat{E_{y}}}{\partial z}\right)+N_{i}^{e} \hat{y} \widehat{E_{y}}+\frac{i k_{y}}{k_{e}^{2}}\left(\frac{\partial N_{i}^{e}}{\partial x} \frac{\partial \widehat{H_{y}}}{\partial z}-\frac{\partial N_{i}^{e}}{\partial z} \frac{\partial \widehat{H_{y}}}{\partial x}\right)\right\} \mathrm{d} x \mathrm{~d} z= \\
& -\sum_{e=1}^{N_{e}} \iint_{D_{e}}\left\{N_{i}^{e} \widehat{J}_{s y}+\frac{i k_{y}}{k_{e}^{2}}\left(\frac{\partial N_{i}^{e}}{\partial x} \widehat{J}_{s x}+\frac{\partial N_{i}^{e}}{\partial z} \widehat{J}_{s z}\right)+\frac{k^{2}}{k_{e}^{2}}\left(\frac{\partial N_{i}^{e}}{\partial z} \widehat{M_{s x}}-\frac{\partial N_{i}^{e}}{\partial x} \widehat{M_{s z}}\right)\right\} d x d z+\sum_{e=1}^{N^{e}} \oint_{\partial D_{e}} N_{i}^{e} \widehat{H_{l}} d l \\
& \sum_{e=1}^{N_{e}} \iint_{D_{e}}\left\{\frac{\partial N_{i}^{e}}{\partial x}\left(\frac{\hat{z}}{k_{e}^{2}} \frac{\partial \widehat{H_{y}}}{\partial x}\right)+\frac{\partial N_{i}^{e}}{\partial z}\left(\frac{\hat{z}}{k_{e}^{2}} \frac{\partial \widehat{H_{y}}}{\partial z}\right)+N_{i}^{e} \hat{z} \widehat{H_{y}}+\frac{i k_{y}}{k_{e}^{2}}\left(-\frac{\partial N_{i}^{e}}{\partial x} \frac{\partial \widehat{E_{y}}}{\partial z}+\frac{\partial N_{i}^{e}}{\partial z} \frac{\partial \widehat{E_{y}}}{\partial x}\right)\right\} \mathrm{d} x \mathrm{~d} z= \\
& -\sum_{e=1}^{N_{e}} \iint_{D_{e}}\left\{N_{i}^{e} \hat{z} \widehat{M_{s y}}+\frac{i k_{y} \hat{z}}{k_{e}^{2}}\left(\frac{\partial N_{i}^{e}}{\partial x} \widehat{M}_{s x}+\frac{\partial N_{i}^{e}}{\partial z} \widehat{M}_{s z}\right)+\frac{\hat{z}}{k_{e}^{2}}\left(\frac{\partial N_{i}^{e}}{\partial z} \widehat{J_{s x}}-\frac{\partial N_{i}^{e}}{\partial x} \widehat{J_{s z}}\right)\right\} \mathrm{d} x \mathrm{~d} z-\sum_{e=1}^{N^{e}} \oint_{\partial D_{e}} N_{i}^{e} \widehat{E}_{l} \mathrm{~d} l
\end{aligned}
$$


where the second integrals on the right hand are line integrals around the boundary $\partial D_{e}$ of element region $D_{e}, \widehat{E}_{l}$ and $\widehat{H}_{l}$ are the tangential components for $\partial D_{e}$, and $N_{e}$ is the total number of the FEM elements.

By setting the above equations numeri cally according to the number of the FEM elements, we formed the global system matrix equation. Because of the continuity of the tangential components $\widehat{E}_{l}$ and $\widehat{H_{l}}$ at the boundary between elements, the last terms on the right hand of equations (7a) and (7b) are canceled and remain only at the boundaries of the discrete area. At these boundaries, according to the simple Dirichlet condition, the field values are assigned to be zero (Druskin and Knizhnerman, 1994).

\subsection{Solution to the linear equations system}

Here, we solved the global system matrix equation with the iterative method. Since the stiffness matrix is symmetric and banded, the incomplete Cholesky preconditioned conjugate gradient (ICCG) method and the modified Cholesky decomposition method were used (Weiss and Newman, 2003). Strictly speaking, the stiffness matrix is simple symmetric, not Hermitian symmetric. However, the conventional ICCG method with simple transpose is effective for such a matrix equation. In this case, ICCG method becomes the incomplete Cholesky preconditioned biconjugate gradient (ICBCG) method (Zhdanov et al, 2007). In the following experiments the ICBCG method (Smith, 1996) was used.

Once $\widehat{E_{y}}$ and $\widehat{H_{y}}$ are obtained, the other components can be obtained from equations (7a) to (7d). These complementary components are calculated from the space derivatives of $\widehat{E_{y}}$ and $\widehat{H}_{y}$ which are evaluated from the space derivatives of the interpolation functions $\partial N^{e}{ }_{i} / \partial x$ and $\partial N^{e}{ }_{i} / \partial z$ in each element. Both of these two space derivatives are most accurate at the corners of the elements in Fig. 2. Whereas the other components obtained from the derivatives of $\widehat{E_{y}}$ and $\widehat{H_{y}}$ show serious errors at points of abrupt variations of $\widehat{E_{y}}$ and $\widehat{H_{y}}$ (Everett and Edwards, 1993).

\subsection{Electromagnetic fields in real space}

The electromagnetic fields in the domain are transformed to the real space $K_{v}$ domain by the inverse Fourier transform. The electromagnetic fields in real space y domain are evaluated by

$$
F\left(x, y=y_{0}, z, \omega\right)=\frac{1}{2 \pi} \int_{-k_{y}}^{k_{y}} \widehat{F}\left(x, k_{y}, z, \omega\right) e^{i k_{y}, y} \mathrm{~d} k_{y},\left(k_{y} \rightarrow \infty\right)
$$

The integral in equation (10) is carried out by cubic spline interpolation of the solutions in the logarithmic wave number domain $K_{y}$ (Constable and Srnka, 2007).

\section{Considerations of the singularity at the source point}

Electric and magnetic dipole sources present singularity at the source location. For instance, in the near zone $(|k r|<<1)$, along strike asymptotic electric field $E_{y}$ generated by a vertical magnetic dipole (VMD) source at the surface of the homogeneous half space is described as follows (Spies and Frischknecht, 1991)

$$
E_{y}=-\frac{M_{s z}}{16 \pi} \frac{x-x_{0}}{r} \omega^{2} \mu^{2} \sigma-i \frac{M_{s z}}{4 \pi} \frac{x-x_{0}}{r^{3}} \omega \mu
$$

where the dipole source is located at $\left(x_{0}, y_{0}, 0\right)$, and the $r=\left[\left(x-x_{0}\right)^{2}+\left(y-y_{0}\right)^{2}\right]^{1 / 2}$ displacement current is neglected. The imaginary part of $E_{y}$ has the singularity of order $O\left(1 / r^{2}\right)$ along the $\mathrm{x}$-axis.

For a horizontal electric dipole (HED) source directed along the strike $y$-axis , the asymptotic electric field $E_{y}$ in the near zone is (Edwards, 2005; Ellingsrud et al, 2002)

$$
E_{y}=-\frac{I d y}{2 \pi \sigma} \frac{1}{r^{3}}-i \frac{I d y}{4 \pi} \frac{\omega \mu}{r}
$$

The real part of $E_{y}$ has the singularity of order $O\left(1 / r^{2}\right)$, and the error of numerical simulation still exists even by using the higher order interpolation function and finer node spacing.

A simple and effective solution to the source singularity is to employ a source which has a finite area. In this study, we adopted the Herrmann's pseudo-delta function (Herrmann, 1979; Furumura and Takenaka, 1996) instead of the delta function.

$$
\begin{gathered}
\delta_{s}\left(x-x_{0}\right)=\frac{1}{2 \tau}\left\{\begin{array}{l}
0 \\
\left(\left(x-x_{0}+2 \tau\right) / \tau\right)^{2} / 2 \\
-\left(\left(x-x_{0}+2 \tau\right) / \tau\right)^{2} / 2+2\left(x-x_{0}+2 \tau\right) / \tau-1 \\
\left(\left(x-x_{0}+2 \tau\right) / \tau\right)^{2} / 2-4\left(x-x_{0}+2 \tau\right) / \tau+8 \\
0
\end{array}\right. \\
\quad\left(x-x_{0}\right) \leq-2 \tau \\
\quad-2 \tau<\left(x-x_{0}\right) \leq-\tau \\
-\tau<\left(x-x_{0}\right) \leq \tau \\
\tau<\left(x-x_{0}\right) \leq 2 \tau \\
2 \tau<\left(x-x_{0}\right)
\end{gathered}
$$

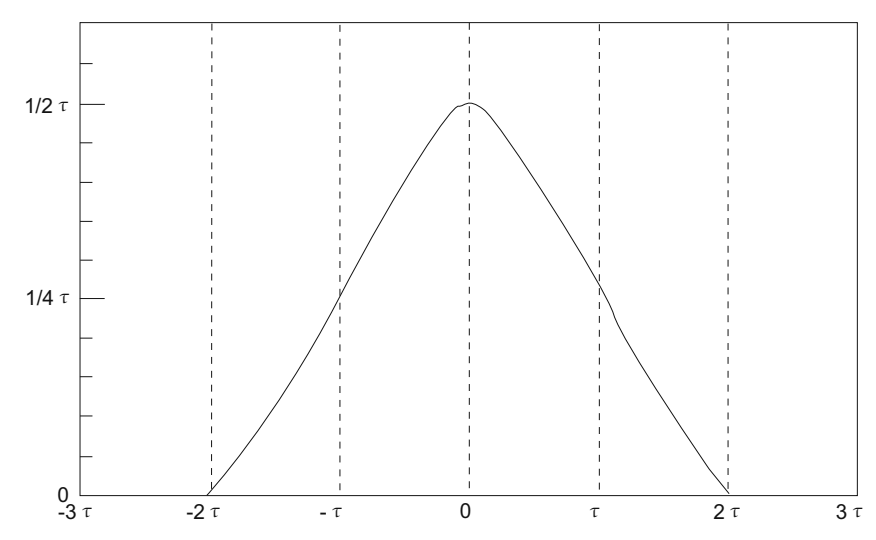

Fig. 3 The spectrum of the pseudo-delta function 
The magnitude and width of the pseudo-delta function depend on parameter $\tau$. By employing Herrmann's pseudodelta function, a three-dimensional pseudo-delta function in our 2.5-D electromagnetic modeling problem was defined as (Unsworth et al, 1993)

$$
\delta_{s}\left(r-r_{0}\right)=\delta_{s}\left(x-x_{0}\right) \delta(y) \delta_{s}\left(z-z_{0}\right)
$$

Here, a true delta function was used for the strike direction since it can be processed by the Fourier transform without difficulty. In the x-z plane, the solution for the dipole source was represented by the smoothed result of the convolution of the true delta function and the pseudo-delta function. The spectrum of the pseudo-delta function is shown in Fig. 3.

\section{Validation of the FEM code}

From equation (10), we know that simulation of the electromagnetic fields needs infinite wave number. However, in our numerical evaluation, we have to select finite number $K_{y}$ of $K_{y}$ to approximate the field variations in $K_{y}$ domain, and the integration in equation (10) is carried out by cubic spline interpolation of the solutions in the wave number $K_{y}$ domain.
In order to validate the code, we checked the numerical result of the electromagnetic field of a specific $K_{y}$ with its analytic solution in homogeneous medium. With respect to the selection of the $K_{y}$, we pursued an optimal range of $K_{y}$ by simulating the homogeneous responses with different range of $K_{y}$ and comparing their analytic solutions.

\subsection{Comparisons with analytic solution in homogeneous medium}

Fig. 4 shows the $\mathrm{Hz}$ and $\mathrm{Hx}$ components of the magnetic field evaluated by the 2.5-D FEM code and their counterparts using the analytic formula (Spies and Habashy, 1995) when $x$ is $67 \mathrm{~m}$ and $y$ is $0 \mathrm{~m}$.

Fig. 5 presents the Ey component of the electric field evaluated by the 2.5-D FEM code and that of the analytic solution when $x$ is $67 \mathrm{~m}$ and $111 \mathrm{~m}$ respectively. Both results show good agreement with analytic solutions, and our code has sound accuracy to simulate the electromagnetic responses. From Fig. 5, it can be seen that as the receivers move far from the source, the field attenuates gradually and our numerical solutions depart from their true values because of inadequate accuracy of the iterative solver.
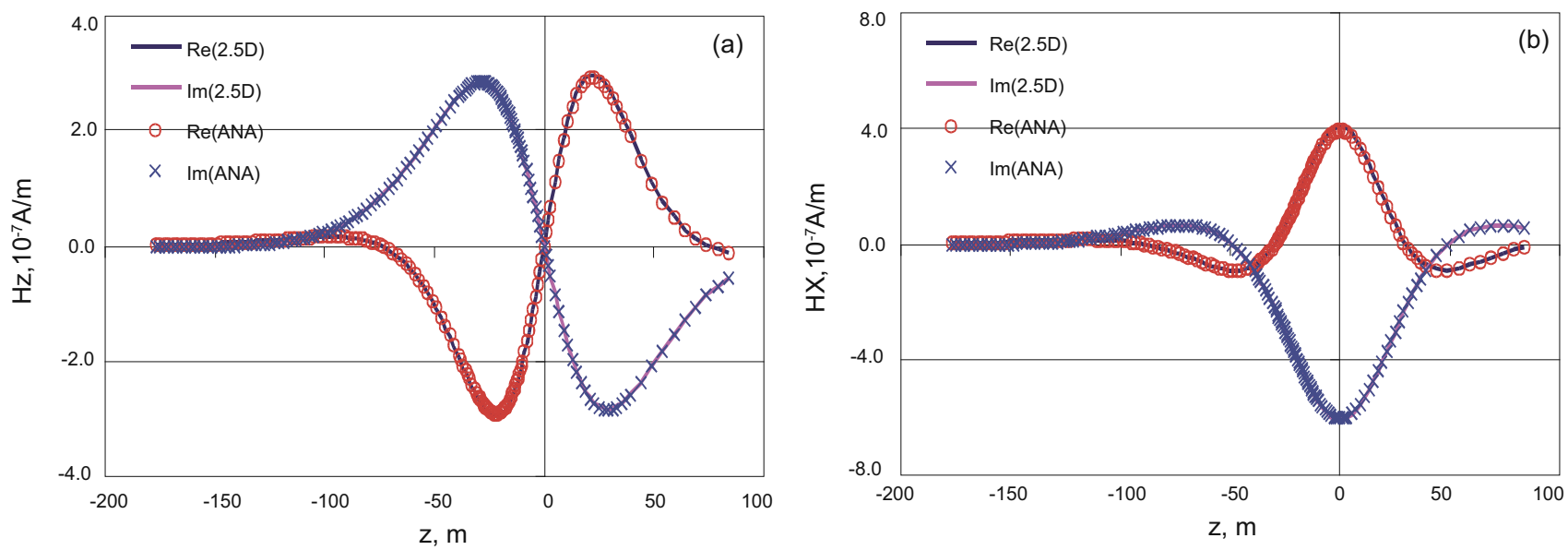

Fig. 4 Comparisons of $\mathrm{Hz}$ (a) and $\mathrm{Hx}$ (b) of the magnetic field evaluated by the 2.5-D FEM code with their counterparts using the analytic equation $(x=67 \mathrm{~m}, y=0 \mathrm{~m})$
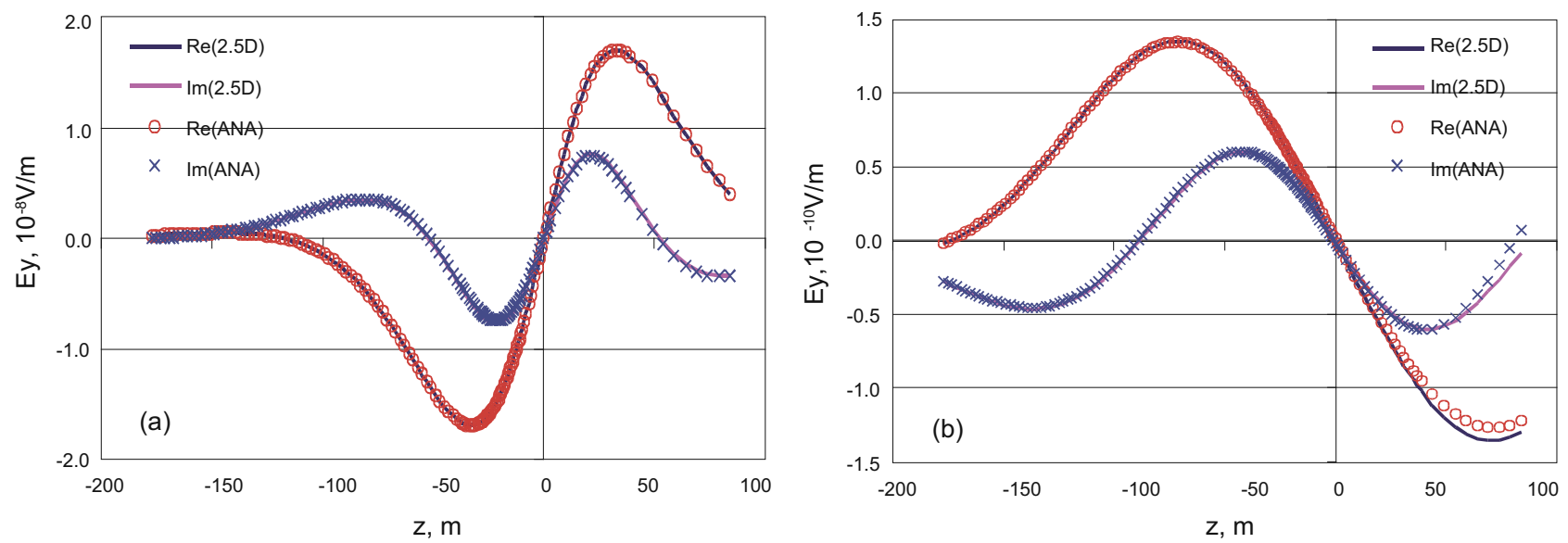

Fig. 5 Comparisons of Ey component of the electric field evaluated by the 2.5-D FEM code with that of the analytic solution when $x=67 \mathrm{~m}$ (a) and $\mathrm{x}=111 \mathrm{~m}$ (b) 


\subsection{Effects of the pseudo-delta function distribution on numerical results}

It is shown that the spectrum is flat within the limit of $K_{x} \times \tau<1$, and as $K_{x}$ increases beyond the limit, it decreases sharply with oscillations. Therefore, the function works as a high-cut filter. Moreover, the Herrmann's function of equation (13) is described by a quadratic curve with respect to the $\mathrm{x}$-coordinate and is thus suitable for the quadratic interpolation function of the FEM. For the dipole source, we have to select an appropriate $\tau$ to accurately simulate the source current distribution.

Fig. 6 shows comparisons of the real and imaginary parts of the magnetic field with that of the analytic solutions for different $\tau$ values. We can know from the numerical results that for a specific distance between source and receiver, $\tau$ has an optimal range which provides accurate numerical results. In our case, the optimal range of $\tau$ is 0.5 to 2.5 .

\subsection{Optimal range of $K_{y}$ values}

In the case of a homogeneous space model, the effective $K_{y}$ range directly depends on the node spacing. To simplify our problem, we consider only $J_{s y}$ in equation (6a) and (6b) and assume that $\left|K_{y}^{2}\right|\left|K^{2}\right|$. The governing differential equation can be reduced to

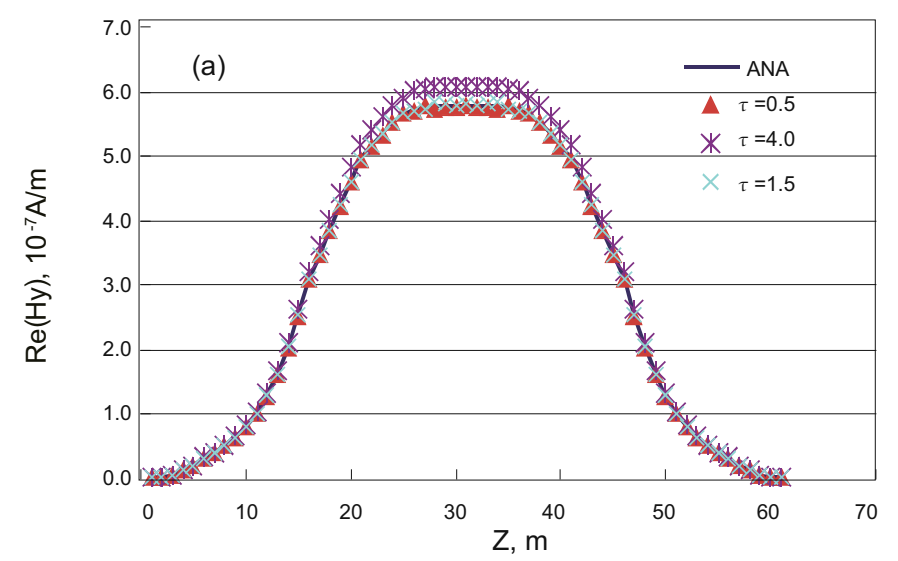

$$
\frac{\partial}{\partial x}\left(\frac{\partial \widehat{E_{y}}}{\partial x}\right)+\frac{\partial}{\partial z}\left(\frac{\partial \widehat{E_{y}}}{\partial z}\right)-k_{y}^{2} \widehat{E_{y}}=\widehat{J_{s y}}
$$

Taking the Fourier transform with respect to $\mathrm{x}$ and $\mathrm{z}$, we obtain

$$
\left(k_{x}^{2}+k_{y}^{2}+k_{z}^{2}\right) \widehat{E_{y}}=-\widehat{J_{s y}}
$$

When $K_{y}^{2} K_{x}^{2}$ and $K_{y}^{2} K_{z}^{2}$, equation (15) no longer depends on the $\mathrm{x}$ and $\mathrm{z}$ coordinates. Therefore, there is a proper upper limit of $K_{y}$ in numerical computations and it should be comparable to the upper limits of $K_{x}$ and $K_{z}$ which depend on space discretization. In numerical evaluation, the effective ranges of $K_{x}$ and $K_{z}$ are definitely smaller than the Nyqusit wave number $K_{N}=\pi / \Delta$, where $\Delta$ is the node spacing. Since the derivatives of the field are included in equation (6a) and (6b), we only need to investigate the accuracy of the derivatives. When we consider the $\mathrm{x}$-derivative of the field, we can assume $f(x)=\exp \left(i k_{x} x\right)$ generally, so the effective wave number $K_{e x}$ is defined as follows (Buell, 1991; Srnka et al, 2006)

$$
f^{\prime}(x)=i k_{e x} \exp \left(i k_{x} x\right)
$$

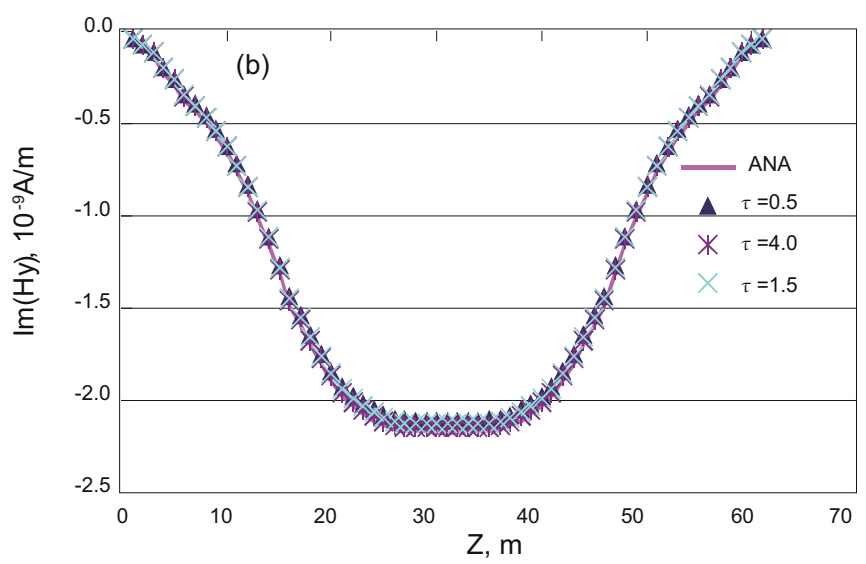

Fig. 6 Comparisons of the simulated magnetic field with that of the analytic solutions (solid line) for different $\tau$ values

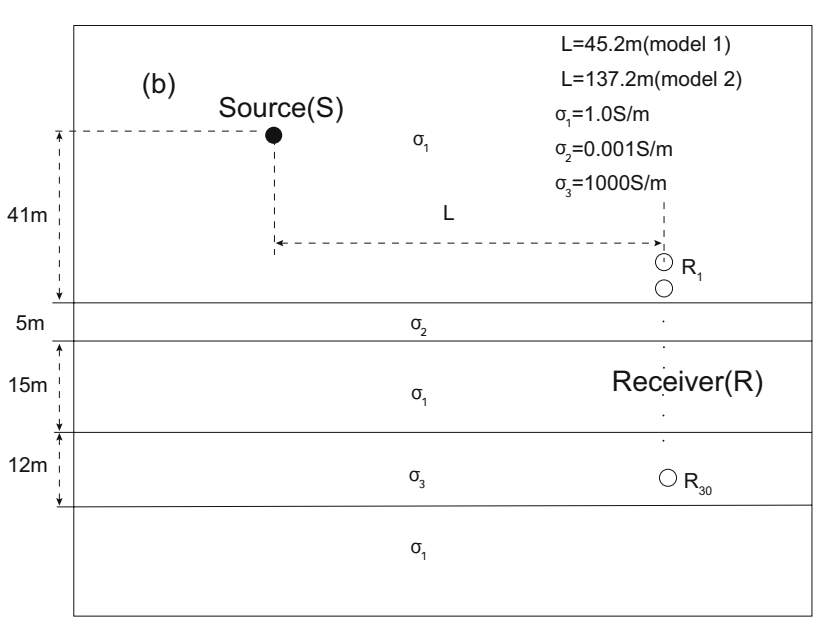

Fig. 7 Five-layer model when $\mathrm{L}=45.2 \mathrm{~m}$
By using the central difference, the effective wave number is given by

$$
k_{e x}=\frac{e^{i k_{x}(x+\Delta)}-e^{i k_{x}(x-\Delta)}}{2 \Delta i e^{i k_{x} x}}=\frac{\sin \left(k_{x} \Delta\right)}{\Delta}
$$

Therefore, the maximum of the effective wave number $K_{e x}$ is $K_{e x}=1 / \Delta$, which is a reasonable value that can determine the effective range. It is advisable to use $K_{e x}$ as a rough threshold of $K_{y}$. Fig. 7 indicates a five-layer model which can be used to address the effects of $K_{y}$ on the numerical results. Fig. 8(a) shows the effects of $K_{y}$ range on the numerical results. In our case, the appropriate range is 0.001 to 1 and the results become deteriorated when we narrow the $K_{y}$ range. Fig. 8(b) shows the effects of different $K_{y}$ on the numerical results. It 

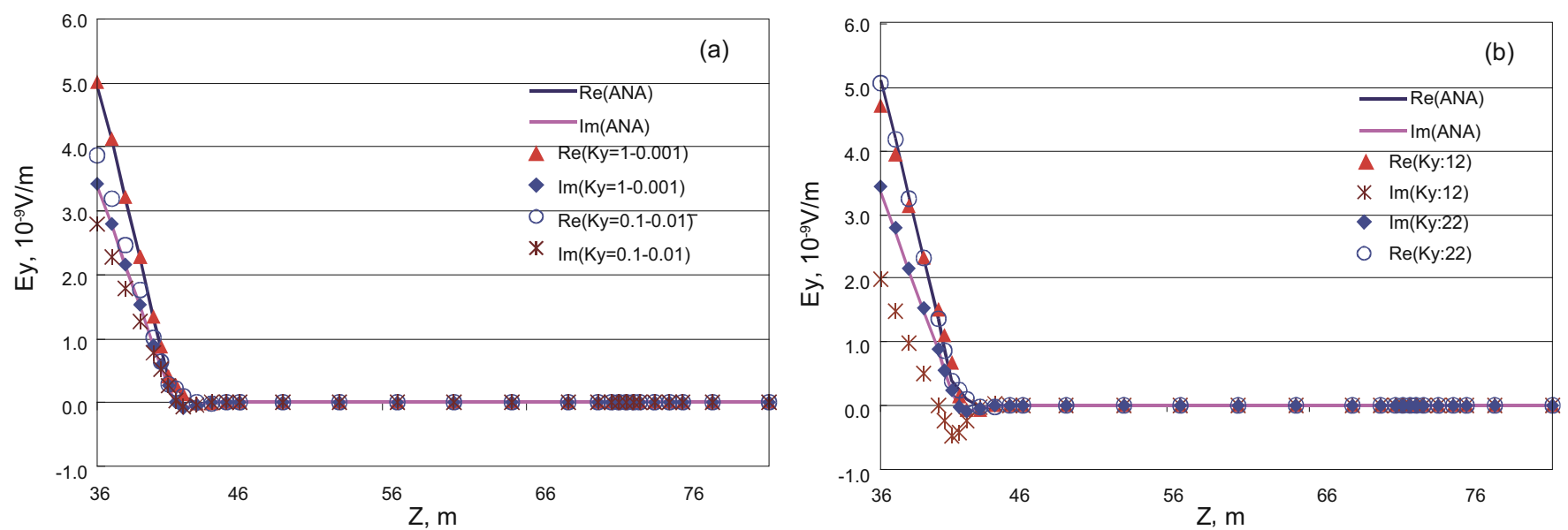

Fig. 8 Comparison of the numerical results with those of the analytic solutions (a) ( $\mathrm{f}=370 \mathrm{~Hz})$ when $\mathrm{Ky} \in(0.001,1.0)$ and $\mathrm{Ky} \in(0.01,1.0)$, and that with different number of Ky values when $\mathrm{Ky} \in(0.001,1.0)$

(b)

is suggested that when we decrease the number of $K_{y}$ to 12 , we cannot obtain an accurate solution of the electromagnetic field. In our numerical modeling, we have to select $K_{y}$ appropriately to ensure accurate results and minimum computing costs.

\section{Simulation of cross-hole electromagnetic responses}

From equations 5(a) to 5(d), we know that other components of the electric and magnetic fields should be calculated from the derivatives of the Ey component of the electric field and the Hy component of the magnetic field. Therefore, the other components will show serious errors at points of abrupt variations of $\widehat{E_{y}}$ and $\widehat{H_{y}}$. Fig. 9(a) provides a model of a deviated bed in a layer background.

Fig. 10 indicates several simulated results with different source locations. From the results of the source one (S1), we can see that at a depth the same as the source, the response presents spikes of error because of the step variations of the conductivity which simulate the slope of the formation. When the source is located within the target zone, the response shows similar features to the horizontal layer model, whereas the responses of the upper and lower sources indicate considerable differences from those of the horizontal layer model. Therefore we can derive the conductivity distribution by comparing with the response of the layer model.

Fig. 11 indicates comparisons of the simulated results of the fault model with different source locations. From the results, we can see that the responses at different locations present pronounced differences. When the source is located below the target zone, the response shows abrupt variations within the fault shift interval. From this anomaly, we can derive the conductivity change by comparison with the response of the layer model.
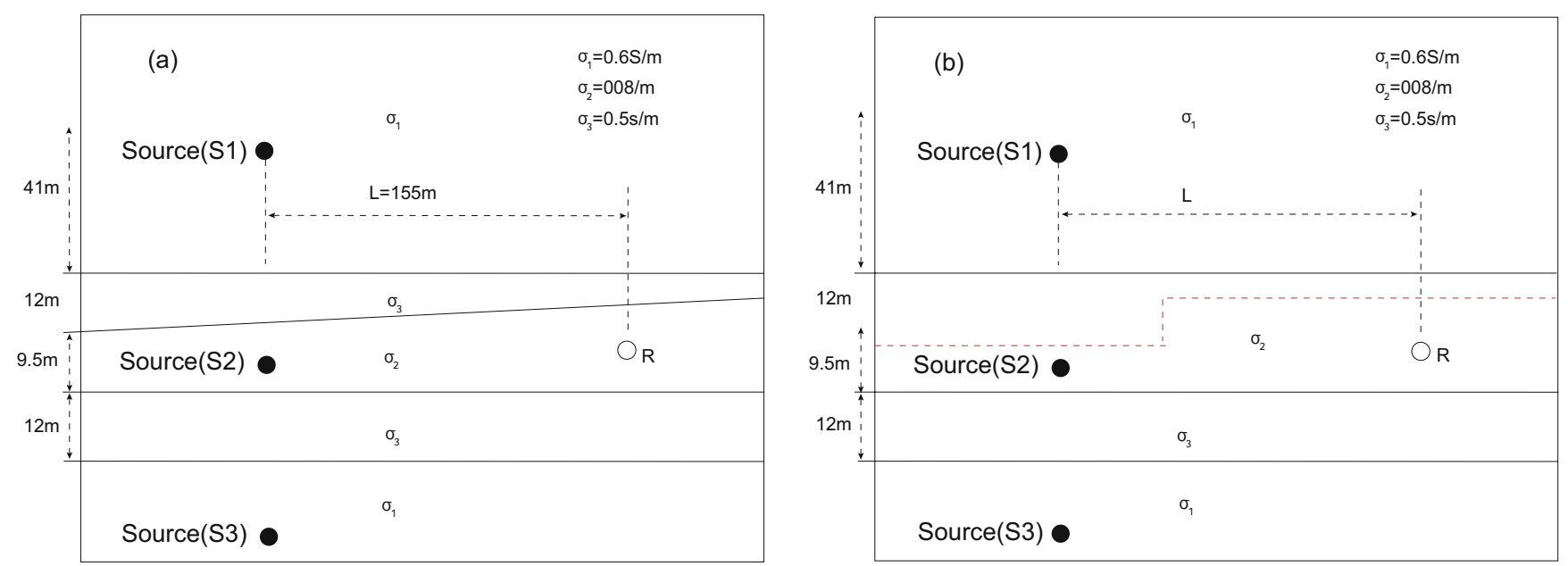

Fig. 9 Deviated layer (a) and fault (b) models to check the electromagnetic responses 

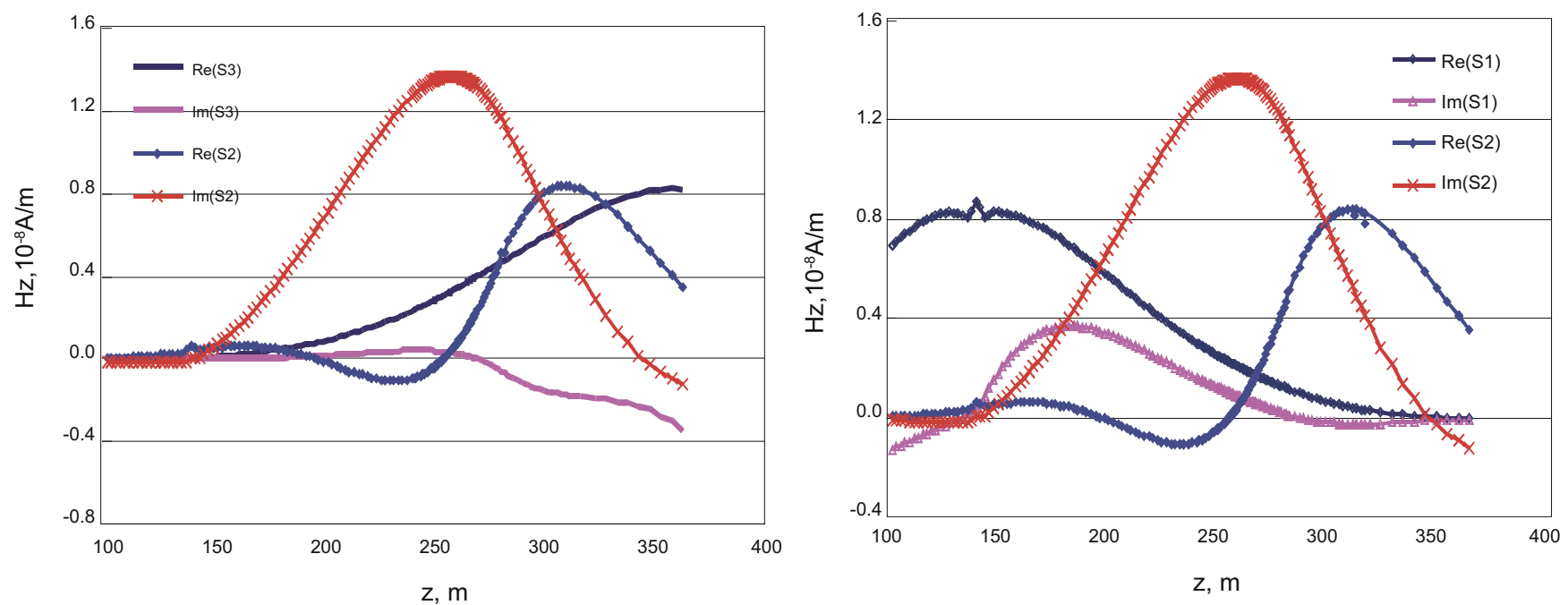

Fig. 10 Electromagnetic responses of the deviated layer when the source in three different locations
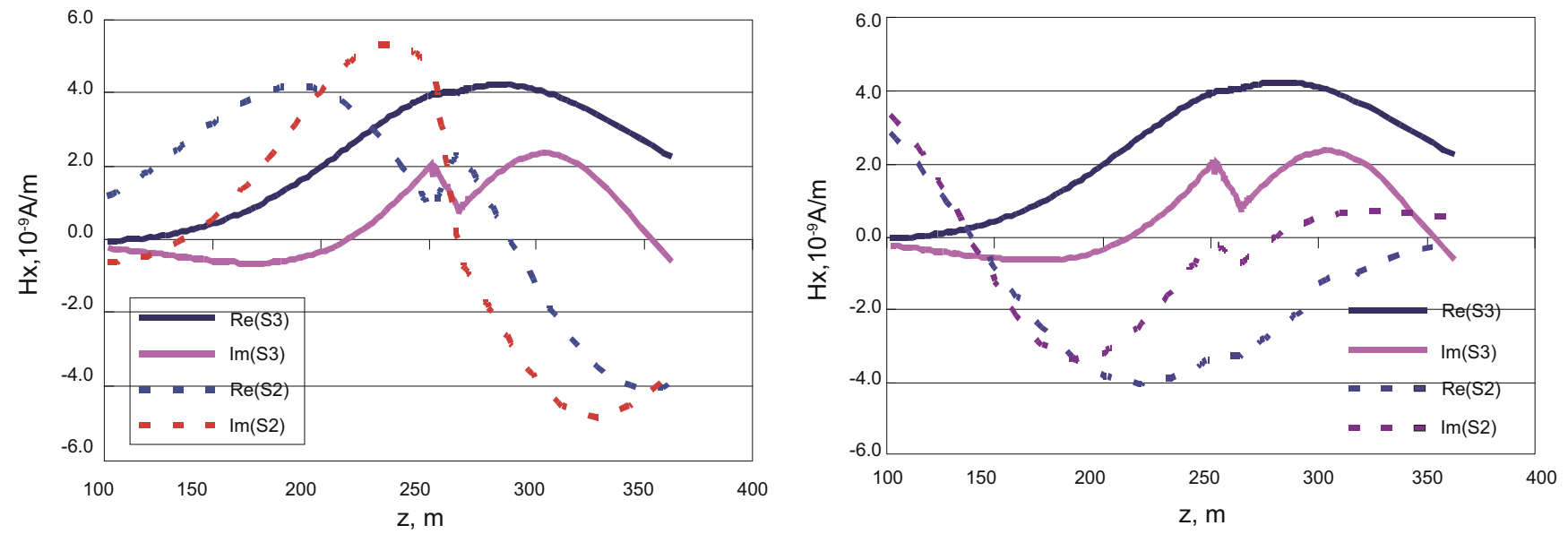

Fig. 11 Electromagnetic responses of the fault model when the source in three different locations

\section{Conclusions}

A method was developed to calculate the electromagnetic responses of a dipole source in 2-D conductive structures. The scheme adopted a pseudo-delta function to distribute the dipole source current and circumvent the singularity problem at the source point. Moreover, isoparametric finite elements were used to accommodate the complex subsurface formation. Numerical results of the homogeneous medium and layer model showed that the pseudo-delta function could approximate the source current satisfactorily when an appropriate $\tau$ value was selected. By properly selecting the y-wave number $\left(K_{\mathrm{y}}\right)$, we could considerably minimize the computing costs by reducing the full three-dimensional problem to a series of two-dimensional problems. Also, within a specific $K_{\mathrm{y}}$ range, the value of $K_{\mathrm{y}}$ had considerable effects on the simulation accuracy. So we have to distribute the $K_{\mathrm{y}}$ value corresponding to the field variations. Simulation results of the slope model and fault model showed that it was possible to derive cross-hole conductivity by comparisons with that of the layer model. Numerical tests showed that our equations could be applied to general complex twodimensional structures containing either an electric or magnetic dipole source in any direction.

\section{References}

Abubakar A., Habashy T., Druskin V., et al. Two-and-half dimensional Forward and Inverse Modeling for Marine CSEM Problems. 76th Ann. Internat. Mtg. Soc. Expl. Geophys. Expanded Abstracts. 2006: 750-754

Avdeeva A., Commer M., and Newman, G. A. Hydrocarbon reservoir detectability study for marine CSEM methods: time domain versus frequency domain, 77th Ann. Internat. Mtg. Soc. Expl. Geophys., Expanded Abstracts. 2007: 628-632 
Buell J C. A hybrid numerical method for three-dimensional spatially developing free shear flows. Journal of Computational Physics. 1991. 95(2): 313-338

Constable K S. and Weiss C. Mapping 3D salt using 2D marine MT: Case study from Gemini Prospect, Gulf of Mexico: 74th Annual International Mtg. Soc. Expl. Geophys., Expanded Abstracts, 2004: 596-599

Constable, S C. Marine electromagnetic methods-Anew tool for offshore exploration: The Leading Edge. 2006. 25: 438-444

Constable S C and Srnka L J. An introduction to marine controlledsource electromagnetic methods for hydrocarbon exploration. Geophysics. 2007.72 (2): WA3-WA12

David L A and Morrison H F. Theoretical and practical considerations for cross well electromagnetic tomography assuming a cylindrical geometry. Geophysics. 1995. 60(3): 846-870

Davydycheva S., Druskin V. and Habashy T. An efficient finitedifference scheme for electromagnetic logging in 3D anisotropic inhomogeneous media. Geophysics. 2003. 68(5): 1525-1536

DruskinV and Knizhnerman L. Spectral approach to solving threedimensional Maxwell's diffusion equations in the time and frequency domains. Radio Science. 1994. 29(4): 937-953

Edwards N. Marine controlled source electromagnetics: Principles and methodologies-future commercial applications: Surveys in Geophysics. 2005. 26(2): 675-700

Ellingsrud S., Eidesmo T., Johansen S., et al. Remote sensing of hydrocarbon layers by seabed logging (SBL): Results from a cruise offshore Angola. The Leading Edge. 2002. 7: 972 - 982

Everett M E and Edwards R N. Transient marine electromagnetics: the 2.5-D forward problem. Geophysical Journal International. 1993. 113(3): 545-561

Farrelly B., Ringstad C., Johnstad S E., et al. Remote characterization of hydrocarbon filled reservoirs at the Troll Field by Sea Bed Logging. EAGE Fall Research Workshop. Rhodes. Greece 19th23rd September. 2004: 662-668

Furumura T and Takenaka H. 2.5-D modeling of elastic waves using the pseudo-spectral method. Geophysical Journal International. 1996. 124: $820-832$

Gallardo L A. and Meju M A. Characterization of heterogeneous nearsurface materials by joint 2D inversion of DC resistivity and seismic data. Geophysical Research Letters. 2003. 30: 1658-1670

Goldman Y, Hubans C, Nicoletis S, et al. A finite element solution for the transient electromagnetic response of an arbitrary two-dimensional resistivity distribution. Geophysics. 1986. 51: 1450-1461

Herrmann R B. SH-wave generation by dislocation source- A numerical study. Bulletin of the Seismological Society of America. 1979. 69(1): $1-15$

Hou J. and Torres-Verdín C. Finite-difference modeling of EM fields using coupled potentials in 3D anisotropic media: Application to borehole logging: 73rd Annual International Mtg., Soc. Expl. Geophys., Expanded Abstracts. 2003: 522-525

Hoversten G M., Gritto R., Washbourne J. et al. Pressure and fluid saturation prediction in a multi-component reservoir, using combined seismic and electromagnetic imaging. Geophysics. 2003. 68(5): 1580 $-1591$

Hoversten G M. and Newman G A. 3D numerical simulation of a deepwater EM exploration survey. 74th Annual International Mtg., Soc. Expl. Geophys., Expanded Abstracts. 2004: 672-675

Hoversten G M., Newman G. A., Geier N., et al. 3D modeling of a deepwater EM exploration survey. Geophysics. 2006. 71(5): G239G248
Johansen S E., Amundsen H E., Rosten T., et al. Subsurface hydrocarbons detected by electromagnetic sounding. First Break. 2005. 23: 31-36

Kong F N., Johnstad S E., Røsten T. et al. A 2.5D finite-element modeling difference method for marine CSEM modeling in stratified anisotropic media. 2008. Geophysics. 73(1): F9-F19

Leppin M. Electromagnetic modeling of 3-D sources over 2-D inhomogeneities in the time domain. Geophysics. 1992. 57(8): 9941003

Liu S X and Sato M. Application of borehole radar for subsurface physical measurement. Journal of Geophysics and Engineering. 2004. 1(3): 221-227

MacGregor L M., Andreis D., Tomlinson J., et al. Controlled-source electromagnetic imaging on the Nuggets-1 reservoir. The Leading Edge. 2006. 25: 984-992

Smith J T. Conservative modeling of 3-D electromagnetic fields, Part2: Biconjugate gradient solution and an accelerator. Geophysics. 1996. 61(5): 1319-1324

Spies B R and Frischknecht F C. Electromagnetic sounding. Electromagnetic methods in applied geophysics: Application. in Nabighian, M. N., Ed., Electromagnetic methods in applied geophysics-theory. 1991. Vol.2: Soc. Explor. Geophys: 431-443

Spies B R and Habashy T M. Sensitivity analysis of cross-well electromagnetics. Geophysics. 1995. 60(3): 834-845

Srnka L J., Carrazone J J., Eriksen E A. et al. Remote reservoir resistivity mapping - An overview. The Leading Edge. 2006. 25: 972-975

Stoyer C H. and Greenfield R J. Numerical solutions of the response of a two-dimensional earth to an oscillating magnetic dipole source, Geophysics. 1976. 41(2): 519-530

Sugeng F, Raiche A, and Xiong Z. Modeling topographic effects using an isoparametric finite element method. The 13th Workshop on Electromagnetic Induction in the Earth. Abstracts. 1996: 109-110

Tompkins M J. Marine controlled source electromagnetic imaging for hydrocarbon exploration: Interpreting subsurface electrical properties. First Break. 2004. 22: 45-51

Ueda T and Zhdanov M S. Fast numerical modeling of marine controlled source electromagnetic data using quasi-liner method. 75th Annual International Mtg., Soc. Expl. Geophys., Expanded Abstracts. 2005: 231-235

Unsworth M J, Travis B J, and Chave A D. Electromagnetic induction by a finite electric dipole source over a 2-D earth. Geophysics. 1993. 58(2): 198-214

Ward S H. and Hohmann G W. Electromagnetic theory for geophysical applications. in Nabighian M. N., Ed., Electromagnetic methods in applied geophysics-theory. Soc. Explor. Geophys. 1988. 1: 131-311

Weiss C J. and Newman G A. Electromagnetic induction in a generalized 3D anisotropic earth, part 2: The LIN preconditioner. Geophysics, 2003. 68(3): 922-930

Zaslavsky M, Davdycheva S., Druskin V. et al. Finite difference solution of the three-dimensional electromagnetic problem using divergence free preconditioners. 76th Ann. Internet. Mtg. Soc. Expl. Geophys., Expanded Abstracts. 2006: 775-778

Zhdanov M.S., Gribenco A. and Cuma M. Regularized focusing inversion of marine CSEM data using minimum vertical support stabilizer, 77th Ann. Internet. Mtg. Soc. Expl. Geophys., Expanded Abstracts. 2007: 579-583

Zienkiewicz O C. The finite element method: third edition. McGrawHill. 1977

(Edited by Yang Lei) 